\title{
Personal Verification/Identification via Analysis of the Peripheral ECG Leads: Influence of the Personal Health Status on the Accuracy
}

\author{
Irena Jekova ${ }^{1}$ and Giovanni Bortolan ${ }^{2}$ \\ ${ }^{1}$ Institute of Biophysics and Biomedical Engineering, Bulgarian Academy of Sciences, Acad. G. Bonchev Street Bl 105, 1113 Sofia, Bulgaria \\ ${ }^{2}$ Institute of Neuroscience (IN), CNR, 35127 Padova, Italy \\ Correspondence should be addressed to Irena Jekova; irena@biomed.bas.bg
}

Received 3 April 2015; Accepted 15 September 2015

Academic Editor: Jason Ng

Copyright ( 2015 I. Jekova and G. Bortolan. This is an open access article distributed under the Creative Commons Attribution License, which permits unrestricted use, distribution, and reproduction in any medium, provided the original work is properly cited.

\begin{abstract}
Traditional means for identity validation (PIN codes, passwords), and physiological and behavioral biometric characteristics (fingerprint, iris, and speech) are susceptible to hacker attacks and/or falsification. This paper presents a method for person verification/identification based on correlation of present-to-previous limb ECG leads: I $\left(r_{\mathrm{I}}\right)$, II $\left(r_{\mathrm{II}}\right)$, calculated from them first principal ECG component $\left(r_{\mathrm{PCA}}\right)$, linear and nonlinear combinations between $r_{\mathrm{I}}, r_{\mathrm{II}}$, and $r_{\mathrm{PCA}}$. For the verification task, the one-toone scenario is applied and threshold values for $r_{\mathrm{I}}, r_{\mathrm{II}}$, and $r_{\mathrm{PCA}}$ and their combinations are derived. The identification task supposes one-to-many scenario and the tested subject is identified according to the maximal correlation with a previously recorded ECG in a database. The population based ECG-ILSA database of 540 patients (147 healthy subjects, 175 patients with cardiac diseases, and 218 with hypertension) has been considered. In addition a common reference PTB dataset (14 healthy individuals) with short time interval between the two acquisitions has been taken into account. The results on ECG-ILSA database were satisfactory with healthy people, and there was not a significant decrease in nonhealthy patients, demonstrating the robustness of the proposed method. With PTB database, the method provides an identification accuracy of $92.9 \%$ and a verification sensitivity and specificity of $100 \%$ and $89.9 \%$.
\end{abstract}

\section{Introduction}

The reliability of automatic person identification has become critical in our life, considering the necessary security for the cases of financial transactions, access control, travelling, and so forth. The traditional means for identity validation, such as PIN codes, passwords, and identity cards, are susceptible to hacker attacks and falsification. In the past few decades, identification based on physiological and behavioral biometric characteristics, such as fingerprint, iris, and speech, were proposed. However, these biometrics could be easily circumvented, for example, by using prosthetic finger or iris [1] or voice playback. Considering these drawbacks, recently the efforts are focused on the development of next generation of biometric characteristics that are internal to the human's body and therefore are robust to the above discussed attacks.
The analysis of the electrocardiogram (ECG) as a biometric tool was started about a decade ago and two general approaches could be distinguished: (i) methods that use measurements after detection of fiducial points and (ii) methods that analyze the overall morphology of the ECG.

The fiducial based approaches had been applied since the very beginning. One of the earliest studies that demonstrated the feasibility of ECG signals for biometrics [2] involved 12 uncorrelated clinical diagnosis features related to $\mathrm{P}, \mathrm{QRS}, \mathrm{T}$ amplitudes, and durations. The interpretation of the similarities/differences between individuals' heartbeats was performed by principal component analysis (PCA) score plots. The authors achieved classification rate of $100 \%$ using 10 of the features. Israel et al. [3] employed 15 temporal features describing the P-QRS-T segment which were fed into a set of discriminant functions for individual recognition. This group 
reported accuracy for the individual classification between $97 \%$ and $100 \%$. In 2008, Wang et al. [4] introduced a two-step detection that incorporates temporal and amplitude measurements based on fiducial points detection and appearance based features that capture the patterns of the heartbeats. The authors achieved $100 \%$ subject identification based on this combined approach.

The methods incorporating time and amplitude characteristics of the heartbeats strongly rely on the correct localization of wave boundaries within the P-QRS-T segment. Current algorithms for ECG delineation are dedicated mainly to the medical applications where the detection of the approximate fiducial point positions is adequate for diagnostic purposes. In contrast, in order to reduce the rejection rate, perfect heartbeat synchronization is required for biometric purposes [5]. For that reason, fiducial independent approaches appeared after 2006. Great part of the proposed methods was based on calculation of correlation coefficients. Plataniotis et al. [6] proposed a method for personal identification applying autocorrelation (AC) of windowed ECG followed by discrete cosine transform (DCT) and reported $100 \%$ accuracy. Agrafioti and Hatzinakos [5] utilized the AC of 5 s ECG segments for biometric identification. The AC signals were processed by discriminant analysis and accuracy of $96.4 \%$ is reported. This work also presented an arrhythmia screening algorithm based on complexity measurement analysis which prevented considering ECG segments with ventricular ectopic beats. Poree et al. [7] reported 100\% identification accuracy using the maximal correlation coefficient applied over 12-lead ECG. The accuracy dropped down to $91.4 \%$ when the method was applied over single ECG lead. Wübbeler et al. [8] formed a two-dimensional heart vector using limb leads ECGs, as well as its first and second temporal derivatives. The proposed identification relies on distance based approach and provides accuracy higher than 97\%. Ye et al. [9] applied discrete wavelet transform (DWT) and independent component analysis (ICA) on ECG beats and obtained 136 features that were further reduced to 26 using PCA. The classification with SVM led to $99.6 \%$ accuracy. Recently, Zokaee and Faez [10] promoted a multimodal biometric system based on ECG and palm print analysis. They used Mel-frequency cepstrum coefficient (MFCC) approach to extract features of ECG biometrics and PCA to extract features from palm print. The accuracy provided by KNN classification was $94.7 \%$. Sidek et al. [11] achieved personal recognition accuracy of $96.1 \%$ by feeding a normalized QRS complex into a Multilayer perceptron. Zhao et al. [1] reported a human ECG identification system based on ECG decomposition in a number of intrinsic mode functions combined with Welch spectral analysis for extraction of significant heartbeat features. PCA was used for feature space reduction. The classification with KNN method provided 95\% identification accuracy.

Despite the reported high accuracy results and the reported evidences for ECG stability in different physiological conditions $[3,7,11,12]$ the validation of ECG for biometric identification requires more severe testing conditions and reduction to easily acquirable ECG leads in order to be adequate to the real situation and to be convenient and reliable for the person under identification process. In this respect, the cited papers have the following limitations:

(1) Several studies use ECG recordings acquired in a very short temporal interval or in the same session $[1,3-$ $5,10,11,13]$ and this produces higher accuracy values. This fact was reported in a recent comparative analysis [14] over 20 authentication methods based on ECG analysis, where significant accuracy degradation was observed when training and testing data come from different sessions if compared to the case of single session.

(2) The proposed methods are applied generally on healthy subjects. However, there are factors of pathologic nature that can severely influence ECG morphology and stability, such as transient or acute cardiac ischemia (manifested in ST-segment changes and sometimes in intra-QRS changes), hypertonia (high ECG voltage), ectopic beats, conductive anomalies causing sometimes intermittent bundle branch blocks, and paroxysmal atrial fibrillation. Many of these changes are of a long-lasting nature and could obviously influence the verification/identification accuracy.

(3) Some studies are based on analysis over all standard 12 ECG leads [7] or on ECG acquired with electrodes placed on the chest [11]. However, the acquisition of the precordial leads is not realistic in many real situations or applications, and the analysis of reduced number of leads produces a reduction of the identification accuracy [7]. The acquisition of ECG for person identification is addressed by Chan et al. [15] and Lourenço et al. [13] who reported 95\% and 94.3\% accuracy using ECG leads acquired from palms and fingers, respectively.

The aim of this paper is to present, test, and validate a method for person verification and identification based on correlation using only the limb ECG leads. The method is developed and tested using a population based ECG database, in which the two recordings have been performed at an interval of 5 years. In addition, the database considers both healthy people and person with some cardiac disease or hypertension. The influence of the presence of nonhealthy patients in the validation phase is studied in detail.

\section{Material and Methods}

2.1. ECG Database. Two independent ECG databases have been used in this study for training and testing the proposed method: a population based database (ECG-ILSA) and a reference database present in many comparisons in literature (the PTB database).

2.1.1. The ECG-ILSA Database. The ECG signals used for training and testing are taken from a computerized ECGILSA database, collected for the Italian Longitudinal Study on Aging Project [16-19]. A random sample of 5632 individuals aged from 65 to 84 years, living independently or in 
institutions, stratified by age and sex with an equal allocation strategy was identified on the demographic lists of the registry office of 8 Italian municipalities. They were followed up with an interval of 5 years in order to study and evaluate physiologic and pathologic modifications connected with aging. The computerized acquisition of ECG signals was performed in about $43 \%$ of the initial population.

This population based ECG-ILSA database consists of 2513 ECG signals in the first phase $\left(T_{1}\right)$ and 1352 ECG signals in the second phase ( $T_{2}=T_{1}+5$ years), and both ECGs are present in 901 patients. For this study, a subset of 540 subjects considered in a previous study [20] was selected. This group is consisting of 147 healthy subjects, 218 people with hypertension, and 175 with cardiac diseases. The healthy group is characterized by absence of cardiovascular and chronic pulmonary disease, no use of drugs that can influence the electrical cardiac activity, and no electrolyte imbalance. The cardiac group is characterized by 56 patients with single diagnosis of cardiovascular diseases, while, in the remaining group with multiple diagnoses, there are 51 patients with MI, 44 with ischemia, and 24 with both.

The ECG recordings are with duration of $10 \mathrm{~s}$ and they include the standard 12 leads, sampled at $500 \mathrm{~Hz}$. In order to have a more robust validation procedure, considering the temporal variability of ECG signal and/or modifications in the pathologies, the learning phase was performed in the healthy group. For this purpose, a random subset of 98 ECGs from the healthy group at times $T_{1}$ and $T_{2}$ represents the training set. Consequently the remaining group of 49 healthy subjects and the entire cardiac and hypertension groups have been considered for the validation/testing procedure.

2.1.2. The Reference PTB Database. We have used an additional test set, the PTB ECG database, which is a common reference database present in the literature for comparative results. The ECG signals are taken from the PhysikalischTechnische Bundesanstalt (PTB) database. The ECGs were collected from healthy volunteers and patients with different heart diseases. The database contains 549 records from 290 subjects, each one represented by one to five recordings, and it includes the conventional 12 leads together with the 3 Frank leads, sampled at $1000 \mathrm{~Hz}$. The testing ECG set used in this study includes 14 healthy control subjects with multiple ECG recordings, for whom the first $\left(T_{1}\right)$ and the last $\left(T_{2}\right)$ ECG recordings have been considered. This dataset is characterized by a short time interval between the two acquisitions at $T_{1}$ and $T_{2}$ : they were performed mainly in a temporal interval from hours (in half of patients) to some months. These ECG recordings have been used in literature as a reference for the evaluation of the methods for person verification/identification $[4,5]$.

2.2. Methods. Aiming at a practicable biometric system, the presented method operates over $10 \mathrm{~s}$ ECG segments and uses only the limb leads I and II. To minimize the negative effect of random noises the ECG signals were passed through

(i) high-pass filter with $0.64 \mathrm{~Hz}$ cutoff frequency to suppress baseline drift, (ii) low-pass filter with cutoff frequency $35 \mathrm{~Hz}$ to reduce muscle noise,

(iii) a notch filter to eliminate power-line interference.

The filtered signals were subjected to QRS detection [21], providing the $\mathrm{R}$-peak positions $\left(\mathrm{QRS}_{\text {index }}\right)$. The $\mathrm{QRS}$ detection was based on comparison of a complex lead, representing the sum of the absolute values of the differentiated lead I and lead II with a combined adaptive threshold

$$
\begin{aligned}
\text { LeadQRS det }(i)= & \operatorname{abs}(\operatorname{LeadI}(i)-\operatorname{LeadI}(i-1)) \\
& +\operatorname{abs}(\operatorname{LeadII}(i)-\operatorname{LeadII}(i-1)) .
\end{aligned}
$$

For the purposes of biometric recognition, an ECG from Set2 (2nd recording) is compared to the ECG in Setl (1st recording) by applying the following procedures:

(1) Calculation of the mean RR interval of the tested $\mathrm{ECG}_{\mathrm{Set} 2}$ and the $\mathrm{ECG}_{\mathrm{Set} 1}$ involved in the current comparison (the smaller RR interval is further referred to as $\left.\mathrm{RR}_{\min }\right)$.

(2) Opening of a window $\left(\mathrm{QRS}_{\text {index }}-\mathrm{RR}_{\min } / 3\right.$ to $\left.\mathrm{QRS}_{\text {index }}+2 \mathrm{RR}_{\min } / 3\right)$ around the QRS complexes detected in $\mathrm{ECG}_{\mathrm{Set} 1}$ and $\mathrm{ECG}_{\mathrm{Set} 2}$.

(3) Application of principle component analysis (PCA) over the opened window, for derivation of combined information for the waveforms in leads I and II.

(4) Calculation of the correlation between each couple $\mathrm{QRS}_{\text {Set1 }}, \mathrm{QRS}_{\mathrm{Set} 2}$, using the equation below:

$$
\begin{aligned}
& r\left(\mathrm{QRS}_{\mathrm{Set} 2}, \mathrm{QRS}_{\mathrm{Set} 1}\right)
\end{aligned}
$$

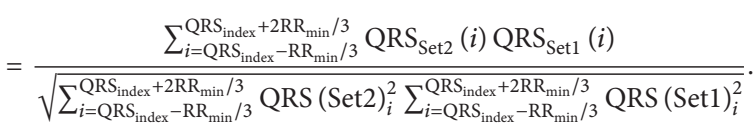

The maximal correlation coefficient representing the best correlated couple $\mathrm{QRS}_{\mathrm{Set} 1}, \mathrm{QRS}_{\mathrm{Set} 2}$ is considered. The values of three independent correlation coefficients $-r_{\text {I }}$ (for the heartbeats in lead I), $r_{\text {II }}$ (lead II), and $r_{\text {PCA }}$ (for the first principal component), two combined correlation coefficients $\left(r_{\mathrm{I}}+r_{\mathrm{II}}\right) / 2,\left(r_{\mathrm{I}}+r_{\mathrm{II}}+r_{\mathrm{PCA}}\right) / 3$, and two nonlinear combinations between them representing the minimal value $\min \left(r_{\mathrm{I}}, r_{\mathrm{II}}, r_{\mathrm{PCA}}\right)$ and the maximal value $\max \left(r_{\mathrm{I}}, r_{\mathrm{II}}, r_{\mathrm{PCA}}\right)$ are further analyzed over the training dataset.

There are two typical scenarios for application of biometric recognition.

(1) Person Verification. The one-to-one scenario is applicable; that is, the ECG of the tested subject in $T_{2}$ is compared to previously recorded ECG in $T_{1}$ with known identity. If the maximal correlation is above a preset threshold value (CorrThr), it is accepted that both ECGs belong to one and the same person and the identity of the tested person is verified.

The accuracy for person verification over the training database is represented by sensitivity (Se_verification) and specificity (Sp_verification). Se_verification is calculated as the percentage of subjects for whom the assessed correlation 


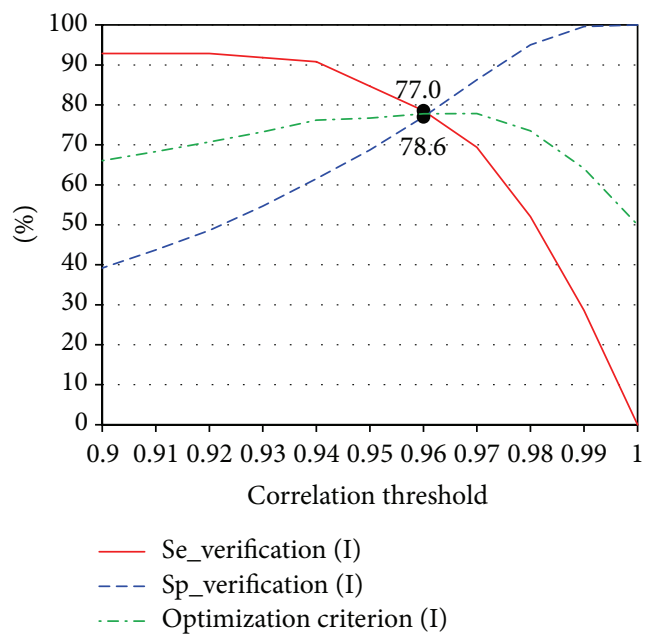

(a)

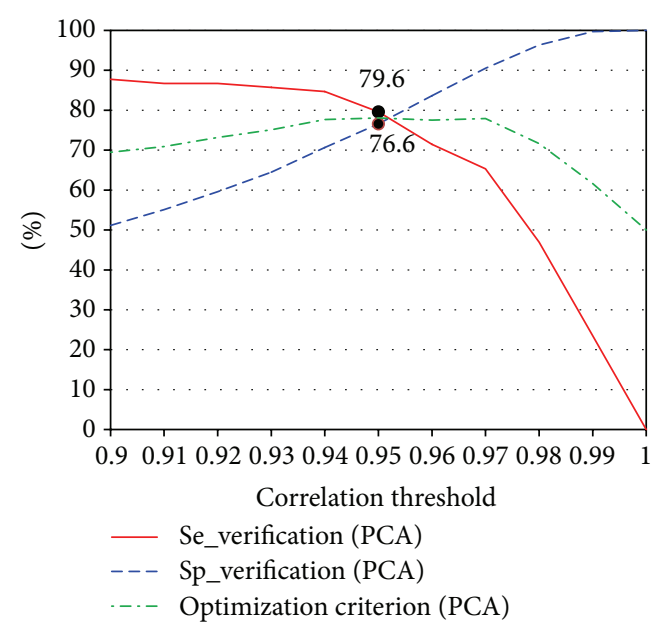

(c)

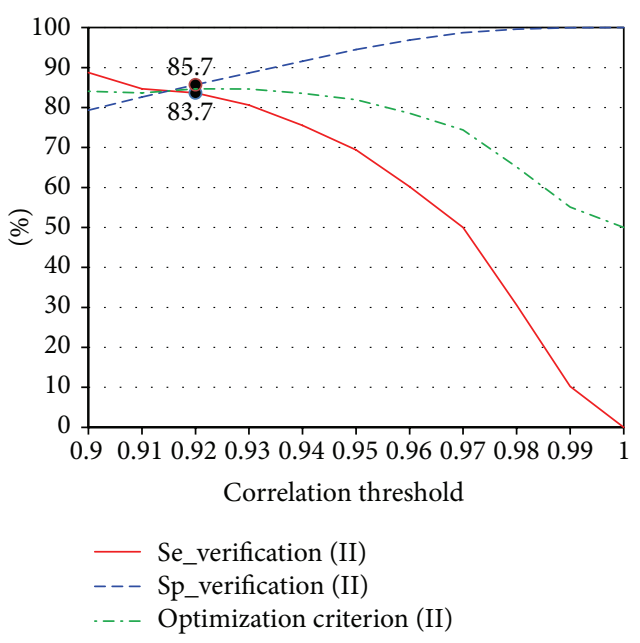

(b)

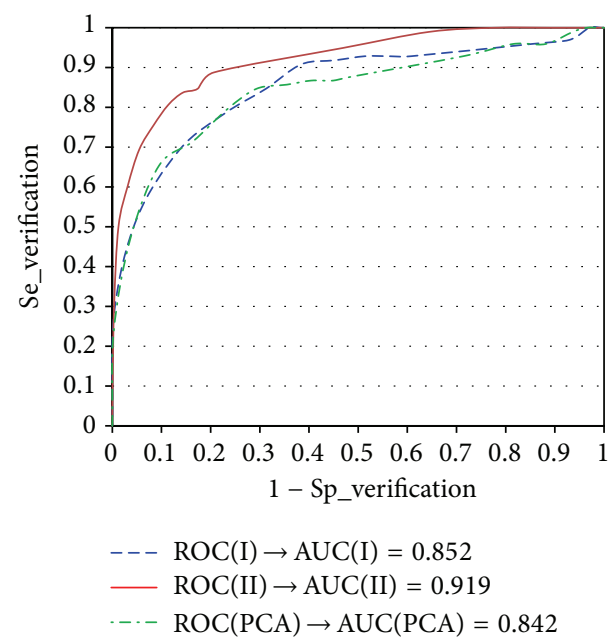

(d)

FIGURE 1: Influence of the threshold for $r_{\mathrm{I}}, r_{\mathrm{II}}$, and $r_{\mathrm{PCA}}$ on the person verification accuracy: (a) Se_verification (I) $=f\left(r_{\mathrm{I}}\right)$; Sp_verification (I) $=f\left(r_{\mathrm{I}}\right)$. (b) Se_verification $(\mathrm{II})=f\left(r_{\mathrm{II}}\right)$; Sp_verification $(\mathrm{II})=f\left(r_{\mathrm{II}}\right)$. (c) Se_verification $(\mathrm{PCA})=f\left(r_{\mathrm{PCA}}\right)$; Sp_verification $($ PCA $)=f\left(r_{\mathrm{PCA}}\right)$. $(\mathrm{d})$ ROC curves and calculated AUC for classification based on $r_{\mathrm{I}}, r_{\mathrm{II}}$, and $r_{\mathrm{PCA}}$. The solid circles mark the optimal solutions.

coefficients and their combinations are above preset threshold values when their ECG signals in $T_{1}$ and $T_{2}$ are compared:

Se_verification

$$
=100 \frac{\sum_{i=1}^{N} r\left(\mathrm{QRS}_{i}\left(T_{2}\right), \mathrm{QRS}_{i}\left(T_{1}\right)\right) \geq \operatorname{CorrThr}}{N},
$$

where $N$ is the number of tested subjects.

Sp_verification is the percentage of cases for which the assessed correlation coefficients and their combinations are below the preset threshold values when comparing ECGs of different subjects:

$$
\begin{aligned}
& \text { Sp_verification }=100 \\
& . \frac{\sum_{i=1}^{N} \sum_{j=1}^{N} r\left(\mathrm{QRS}_{i}\left(T_{2}\right), \mathrm{QRS}_{j}\left(T_{1}\right)\right)<\text { CorrThr }}{N\left(N_{\text {total }}-1\right)}
\end{aligned}
$$

where $N$ is the number of tested subjects and $N_{\text {total }}$ is the number of subjects in the database for comparison.

Threshold values for $r_{\mathrm{I}}, r_{\mathrm{II}}, r_{\mathrm{PCA}},\left(r_{\mathrm{I}}+r_{\mathrm{II}}\right) / 2,\left(r_{\mathrm{I}}+r_{\mathrm{II}}+\right.$ $\left.r_{\mathrm{PCA}}\right) / 3, \max \left(r_{\mathrm{I}}, r_{\mathrm{II}}, r_{\mathrm{PCA}}\right)$, and $\min \left(r_{\mathrm{I}}, r_{\mathrm{II}}, r_{\mathrm{PCA}}\right)$ are selected, based on analysis of the relation between their values and the verification accuracy (see Figures 1(a)-1(c) and 2(a)-

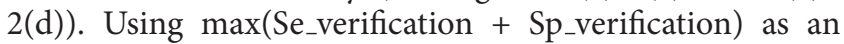
optimization criterion, we selected threshold values for $r_{\mathrm{I}}$ (0.96), $r_{\mathrm{II}}(0.92), r_{\mathrm{PCA}}(0.95),\left(r_{\mathrm{I}}+r_{\mathrm{II}}\right) / 2(0.93),\left(r_{\mathrm{I}}+r_{\mathrm{II}}+\right.$ $\left.r_{\mathrm{PCA}}\right) / 3(0.94), \max \left(r_{\mathrm{I}}, r_{\mathrm{II}}, r_{\mathrm{PCA}}\right)(0.97)$, and $\min \left(r_{\mathrm{I}}, r_{\mathrm{II}}, r_{\mathrm{PCA}}\right)$ (0.93). The achieved accuracy indices are presented in Table 1. Receiver operating characteristic (ROC) curves are built and the area under the curve (AUC) is calculated for $r_{\mathrm{I}}, r_{\mathrm{II}}$, $r_{\mathrm{PCA}},\left(r_{\mathrm{I}}+r_{\mathrm{II}}\right) / 2,\left(r_{\mathrm{I}}+r_{\mathrm{II}}+r_{\mathrm{PCA}}\right) / 3, \max \left(r_{\mathrm{I}}, r_{\mathrm{II}}, r_{\mathrm{PCA}}\right)$, and $\min \left(r_{\mathrm{I}}, r_{\mathrm{II}}, r_{\mathrm{PCA}}\right)$. AUC could be used for scoring the potential for person verification of different models.

(2) Person Identification. The one-to-many scenario is applicable to a specific group of persons. The ECG in $T_{2}$ of 


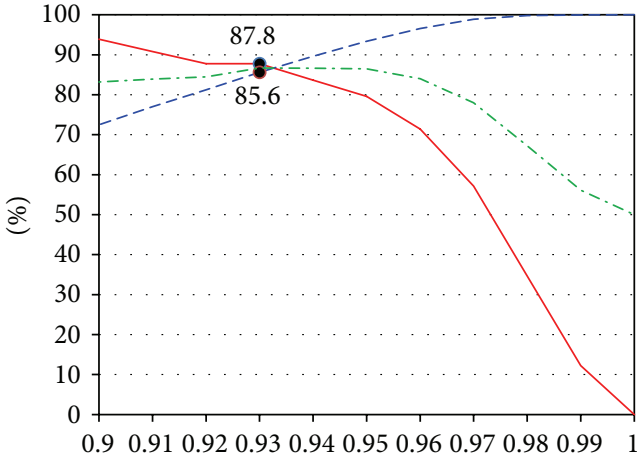

Correlation threshold

- Se_verification (I + II)

--- Sp_verification $(\mathrm{I}+\mathrm{II})$

-.-- Optimization criterion (I + II)

(a)

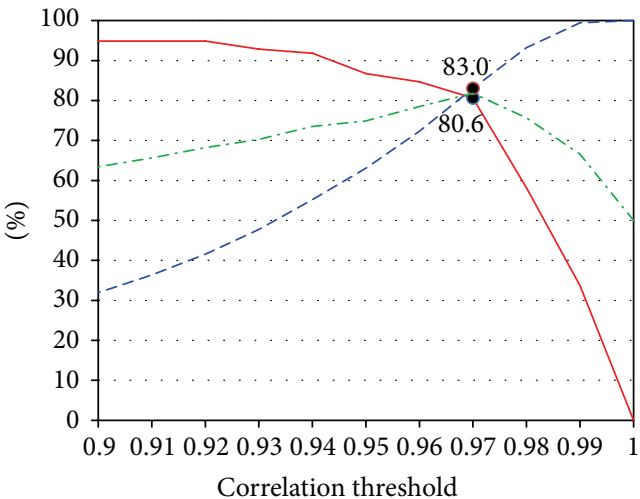

- Se_verification $(\max (I$, II, PCA $))$

- - Sp_verification $(\max (I, I I, P C A))$

...- Optimization criterion $(\max (\mathrm{I}, \mathrm{II}, \mathrm{PCA}))$

(c)

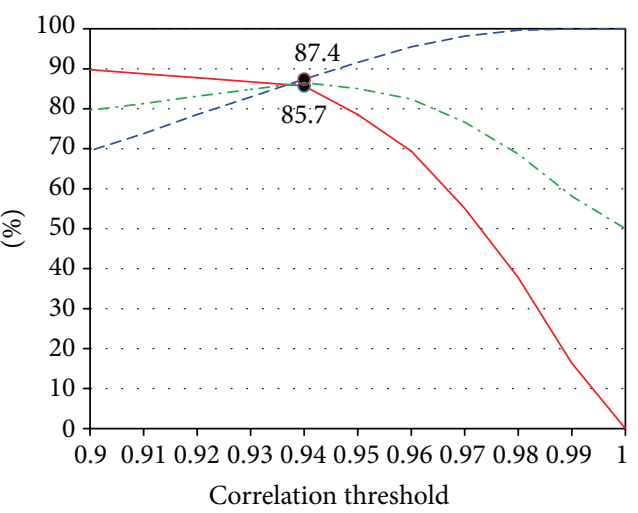

_ Se_verification (I + II + PCA)

- - Sp_verification $(\mathrm{I}+\mathrm{II}+\mathrm{PCA})$

-.- Optimization criterion (I + II + PCA)

(b)

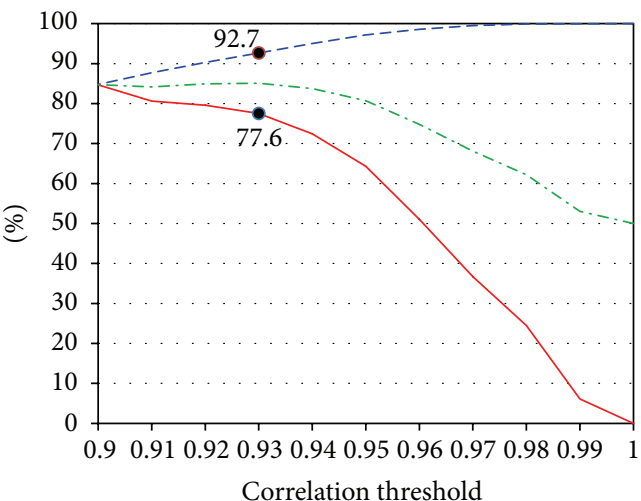

Correlation threshold

- Se_verification (min(I, II, PCA))

- - Sp_verification (min(I, II, PCA))

.... Optimization criterion (min(I, II, PCA))

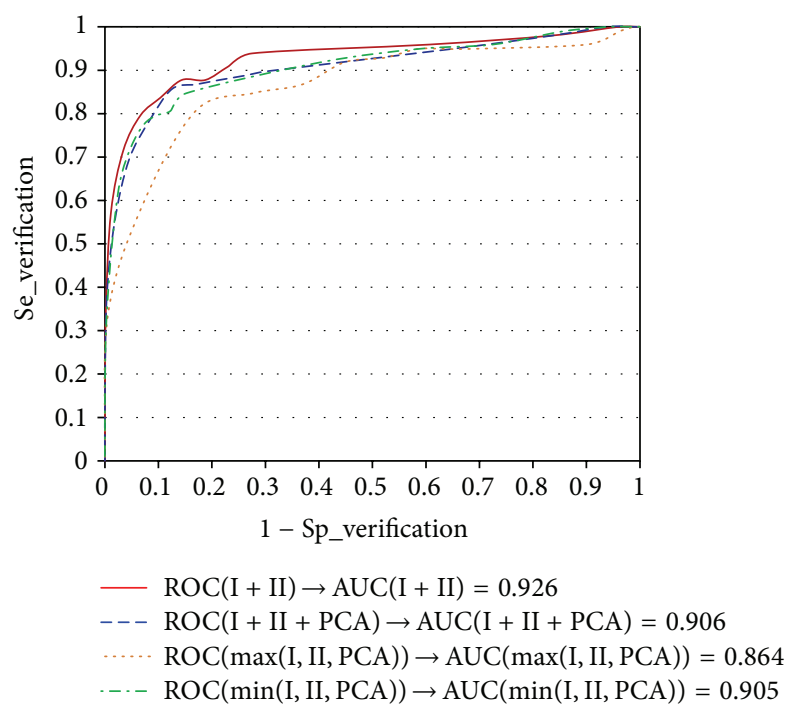

(e)

FIGURE 2: Influence of the threshold for $\left(r_{\mathrm{I}}+r_{\mathrm{II}}\right) / 2,\left(r_{\mathrm{I}}+r_{\mathrm{II}}+r_{\mathrm{PCA}}\right) / 3, \max \left(r_{\mathrm{I}}, r_{\mathrm{II}}, r_{\mathrm{PCA}}\right)$, and $\min \left(r_{\mathrm{I}}, r_{\mathrm{II}}, r_{\mathrm{PCA}}\right)$ on the person verification accuracy: (a) Se_verification (I + II $)=f\left(\left(r_{\mathrm{I}}+r_{\mathrm{II}}\right) / 2\right)$; Sp_verification $(\mathrm{I}+\mathrm{II})=f\left(\left(r_{\mathrm{I}}+r_{\mathrm{II}}\right) / 2\right)$. (b) Se_verification $(\mathrm{I}+\mathrm{II}+\mathrm{PCA})=f\left(\left(r_{\mathrm{I}}+r_{\mathrm{II}}+\right.\right.$ $\left.\left.r_{\mathrm{PCA}}\right) / 3\right)$; Sp_verification (I + II + PCA $)=f\left(\left(r_{\mathrm{I}}+r_{\mathrm{II}}+r_{\mathrm{PCA}}\right) / 3\right)$. (c) Se_verification $\left(\max \left(r_{\mathrm{I}}, r_{\mathrm{II}}, r_{\mathrm{PCA}}\right)\right)=f\left(\max \left(r_{\mathrm{I}}, r_{\mathrm{II}}, r_{\mathrm{PCA}}\right)\right)$; Sp_verification $\left(\max \left(r_{\mathrm{I}}, r_{\mathrm{II}}, r_{\mathrm{PCA}}\right)\right)=f\left(\max \left(r_{\mathrm{I}}, r_{\mathrm{II}}, r_{\mathrm{PCA}}\right)\right)$. (d) Se_verification $\left(\min \left(r_{\mathrm{I}}, r_{\mathrm{II}}, r_{\mathrm{PCA}}\right)\right)=f\left(\min \left(r_{\mathrm{I}}, r_{\mathrm{II}}, r_{\mathrm{PCA}}\right)\right) ; \operatorname{Sp}$-verification $\left(\min \left(r_{\mathrm{I}}, r_{\mathrm{II}}, r_{\mathrm{PCA}}\right)\right)=$ $f\left(\min \left(r_{\mathrm{I}}, r_{\mathrm{II}}, r_{\mathrm{PCA}}\right)\right)$. (e) ROC curves and calculated AUC for classification based on $r_{\mathrm{I}}+r_{\mathrm{II}}, r_{\mathrm{I}}+r_{\mathrm{II}}+r_{\mathrm{PCA}}, \min \left(r_{\mathrm{I}}, r_{\mathrm{II}}, r_{\mathrm{PCA}}\right)$, and $\max \left(r_{\mathrm{I}}, r_{\mathrm{II}}\right.$, $\left.r_{\mathrm{PCA}}\right)$. The solid circles mark the optimal solutions. 
TABLE 1: Verification/identification accuracy calculated over the training database: selected threshold value (VerTHR), sensitivity, specificity, and value of the optimization criterion for person verification; accuracy for person identification (AccID), $N=98$.

\begin{tabular}{|c|c|c|c|c|c|}
\hline & VerTHR & Se_verification & Sp_verification & Opt. criterion & AccID \\
\hline$r_{\mathrm{I}}$ & 0.96 & $78.6 \%(77 / 98)$ & $77 \%(7320 / 9506)$ & $77.8 \%$ & $53.1 \%(52 / 98)$ \\
\hline$r_{\mathrm{II}}$ & 0.92 & $83.7 \%(82 / 98)$ & $85.7 \%(8147 / 9506)$ & $84.7 \%$ & $59.2 \%(58 / 98)$ \\
\hline$r_{\mathrm{PCA}}$ & 0.95 & $79.6 \%(78 / 98)$ & $76.6 \%(7282 / 9506)$ & $78.1 \%$ & $52 \%(51 / 98)$ \\
\hline$\left(r_{\mathrm{I}}+r_{\mathrm{II}}\right) / 2$ & 0.93 & $\mathbf{8 7 . 8} \%(86 / 98)$ & $\mathbf{8 6 . 6} \%(8232 / 9506)$ & $86.7 \%$ & $\mathbf{6 9 . 4} \%(68 / 98)$ \\
\hline$\left(r_{\mathrm{I}}+r_{\mathrm{II}}+r_{\mathrm{PCA}}\right) / 3$ & 0.94 & $\mathbf{8 5 . 7 \%}(84 / 98)$ & $\mathbf{8 7 . 4} \%(8308 / 9506)$ & $86.6 \%$ & $71.4 \%(70 / 98)$ \\
\hline $\max \left(r_{\mathrm{I}}, r_{\mathrm{II}}, r_{\mathrm{PCA}}\right)$ & 0.97 & $80.6 \%(79 / 98)$ & $83.0 \%(7890 / 9506)$ & $81.8 \%$ & $59.2 \%(58 / 98)$ \\
\hline $\min \left(r_{\mathrm{I}}, r_{\mathrm{II}}, r_{\mathrm{PCA}}\right)$ & 0.93 & $77.6 \%(76 / 98)$ & $92.7 \%(8812 / 9506)$ & $85.1 \%$ & $65.3 \%(64 / 98)$ \\
\hline
\end{tabular}

the subject under identity examination is compared to all previously recorded ECG in $T_{1}$ and the maximal correlation of this comparison detects the identity to the tested subject. The identification accuracy (AccID) is calculated as the percentage of subjects for whom ECG in $T_{2}$ is maximally correlated with their own ECG in $T_{1}$.

\section{Results}

The Se_verification, Sp_verification, and the value of the optimization criterion achieved for the training database with the selected thresholds for the $r_{\mathrm{I}}, r_{\mathrm{II}}, r_{\mathrm{PCA}}$, and their combinations are presented in Table 1. The accuracy for person identification, calculated as the percentage of subjects, whose ECG in $T_{2}$ is maximally correlated with their own ECG in $T_{1}$ is also presented in Table 1.

The proposed method for person verification/identification was independently tested over the two test databases. For the verification task, the correlation coefficients threshold values observed over the training database were applied. Aiming to obtain comparable results to the one reported in literature and to provide an unbiased basis for assessment of the influence of the personal health status on the verification/identification accuracy, we performed 2 types of tests as follows:

(i) Tests include only the healthy subjects: the results are presented in Table 2 for ILSA test dataset (49 persons) and Table 4 for PTB test dataset (14 persons). Figure 3 illustrates the ROC curves with the respective AUCs that prove the similar behavior of the designed person validation method over the training and test ILSA datasets.

(ii) Tests include all patients from the ILSA test dataset (442 persons): the results for verification and identification accuracy are presented in Table 3, considering the three groups of patients: healthy, cardiac, and hypertension ones.

Examples that show cases of both correct person verification and identification are presented in Figure 4 for the training database and Figure 5 for the publicly available PTB test dataset. Despite the strong correlations observed in both examples, it is visible that there is a complete matching between the waveforms of the ECGs in Figure 5, while the
TABLE 2: Sensitivity and specificity for person verification and accuracy for person identification obtained for the healthy subset in the test ILSA dataset ( $N=49$ subjects).

\begin{tabular}{lccc}
\hline & Se_verification & Sp_verification & AccID \\
\hline$r_{\mathrm{I}}$ & $77.6 \%(38 / 49)$ & $83 \%$ & $63.3 \%$ \\
& & $(1912 / 2304)$ & $(31 / 49)$ \\
$r_{\mathrm{II}}$ & $87.8 \%(43 / 49)$ & $81.7 \%$ & $61.2 \%$ \\
& & $(1882 / 2304)$ & $(30 / 49)$ \\
$r_{\mathrm{PCA}}$ & $81.6 \%(40 / 49)$ & $80.7 \%$ & $59.2 \%$ \\
& & $(1859 / 2304)$ & $(29 / 49)$ \\
$\left(r_{\mathrm{I}}+r_{\mathrm{II}}\right) / 2$ & $\mathbf{8 9 . 8} \%(44 / 49)$ & $\mathbf{8 3 . 9} \%$ & $\mathbf{7 7 . 6} \%$ \\
& & $(1933 / 2304)$ & $(38 / 49)$ \\
$\left(r_{\mathrm{I}}+r_{\mathrm{II}}+r_{\mathrm{PCA}}\right) / 3$ & $\mathbf{8 7 . 8} \%(43 / 49)$ & $\mathbf{8 6 . 6} \%$ & $\mathbf{7 5 . 5} \%$ \\
$\max \left(r_{\mathrm{I}}, r_{\mathrm{II}}, r_{\mathrm{PCA}}\right)$ & $75.5 \%(37 / 49)$ & $87.5 \%$ & $65.3 \%$ \\
& & $(2016 / 2304)$ & $(32 / 49)$ \\
$\min \left(r_{\mathrm{I}}, r_{\mathrm{II}}, r_{\mathrm{PCA}}\right)$ & $79.6 \%(39 / 49)$ & $90.6 \%$ & $75.5 \%$ \\
& & $(2087 / 2304)$ & $(37 / 49)$ \\
\hline
\end{tabular}

ECGs in Figure 4 show slight changes mainly in the QRS amplitudes.

Figures 6 and 8 present cases for which neither the verification nor the identification will be successful. The example in Figure 7 illustrates a case, which will be correctly verified if $r_{\mathrm{II}}, r_{\mathrm{PCA}}$, or the combined correlation coefficients are used and erroneously rejected if $r_{\mathrm{I}}<0.96$ is applied for verification. The only chance this person to be correctly identified is the application of one of the combined correlation coefficients $\left(r_{\mathrm{I}}+r_{\mathrm{II}}\right) / 2$ and $\left(r_{\mathrm{I}}+r_{\mathrm{II}}+r_{\mathrm{PCA}}\right) / 3$.

\section{Discussion}

This paper presents a method for person verification and identification based on cross-correlation over ECG signals. Aiming to assure a convenient and a comfortable acquisition procedure for the tested person, the proposed algorithm uses only the independent limb leads I, II.

The person verification is performed by comparison between the ECG of the person who pretends for certain identity and a previously recorded ECG of subject with the tested identity, using the maximal cross-correlation as an estimator for their similarity. The curves in Figures 1(a)-1(c) and $2(a)-2(d)$ provide the opportunity to select threshold 
TABLE 3: Sensitivity and specificity for person verification, together with the accuracy for person identification, obtained for the entire test ILSA dataset ( $N=442$ subjects), considering the health status of the tested subjects.

\begin{tabular}{|c|c|c|c|c|c|c|c|c|c|}
\hline & \multicolumn{3}{|c|}{ Se_verification (\%) } & \multicolumn{3}{|c|}{ Sp_verification (\%) } & \multicolumn{3}{|c|}{ AccID (\%) } \\
\hline & Healthy & Card & Hypt & Healthy & Card & Hypt & Healthy & Card & Hypt \\
\hline$r_{\text {I }}$ & $77.6 \%$ & $74.9 \%$ & $76.1 \%$ & $82.8 \%$ & $87.9 \%$ & $81.4 \%$ & $38.8 \%$ & $46.3 \%$ & $46.3 \%$ \\
\hline$r_{\text {II }}$ & $87.8 \%$ & $77.7 \%$ & $78.9 \%$ & $85.4 \%$ & $90.8 \%$ & $86.7 \%$ & $42.9 \%$ & $52.0 \%$ & $44.5 \%$ \\
\hline$r_{\mathrm{PCA}}$ & $81.6 \%$ & $80.0 \%$ & $79.4 \%$ & $81.6 \%$ & $85.0 \%$ & $78.9 \%$ & $42.9 \%$ & $48.0 \%$ & $41.3 \%$ \\
\hline$\left(r_{\mathrm{I}}+r_{\mathrm{II}}\right) / 2$ & $89.8 \%$ & $86.3 \%$ & $82.6 \%$ & $86.6 \%$ & $91.8 \%$ & $\mathbf{8 7 . 0} \%$ & $59.2 \%$ & $60.6 \%$ & $53.7 \%$ \\
\hline$\left(r_{\mathrm{I}}+r_{\mathrm{II}}+r_{\mathrm{PCA}}\right) / 3$ & $\mathbf{8 7 . 8} \%$ & $78.3 \%$ & $79.8 \%$ & $\mathbf{8 8 . 9} \%$ & $92.9 \%$ & $88.4 \%$ & $61.2 \%$ & $62.3 \%$ & $54.6 \%$ \\
\hline $\max \left(r_{\mathrm{I}}, r_{\mathrm{II}}, r_{\mathrm{PCA}}\right)$ & $75.5 \%$ & $73.1 \%$ & $74.8 \%$ & $87.4 \%$ & $90.9 \%$ & $85.8 \%$ & $42.9 \%$ & $57.7 \%$ & $46.8 \%$ \\
\hline $\min \left(r_{\mathrm{I}}, r_{\mathrm{II}}, r_{\mathrm{PCA}}\right)$ & $79.6 \%$ & $69.7 \%$ & $71.6 \%$ & $92.6 \%$ & $95.6 \%$ & $92.9 \%$ & $59.2 \%$ & $54.9 \%$ & $49.5 \%$ \\
\hline
\end{tabular}
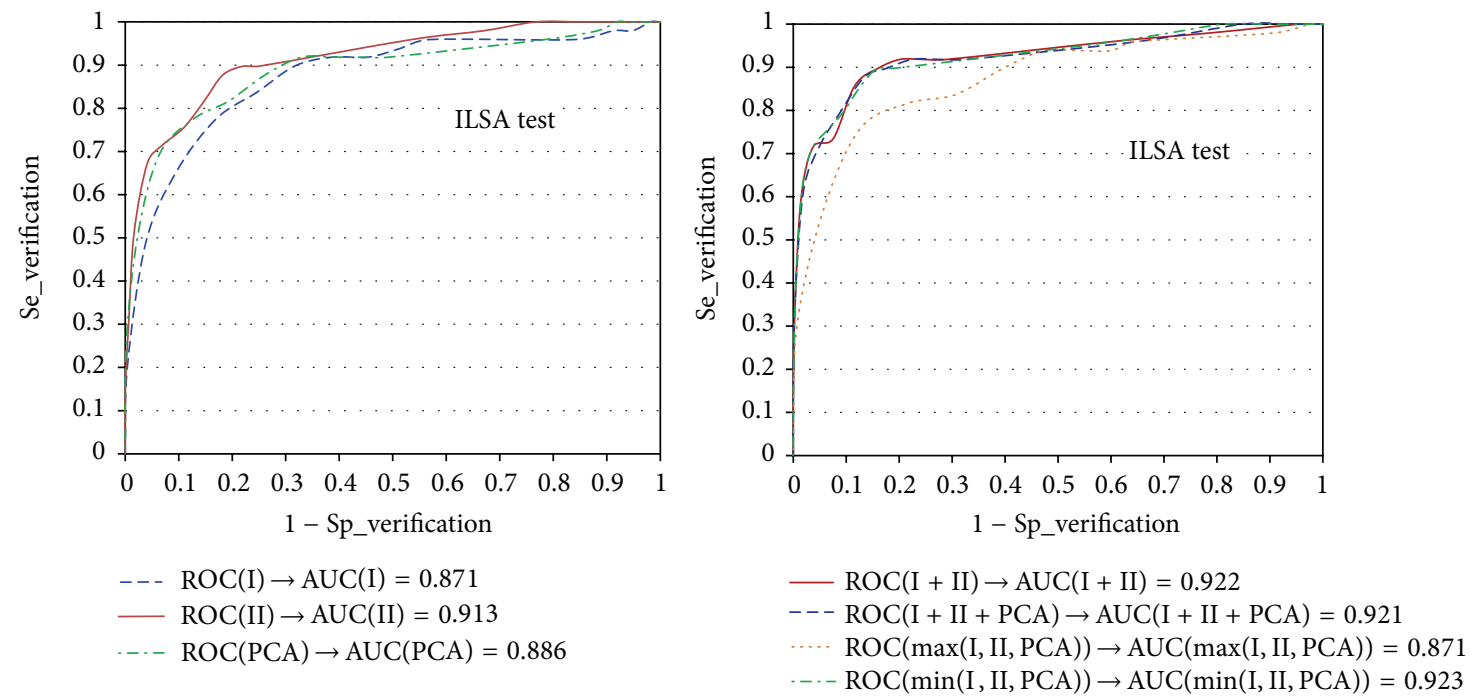

(a)
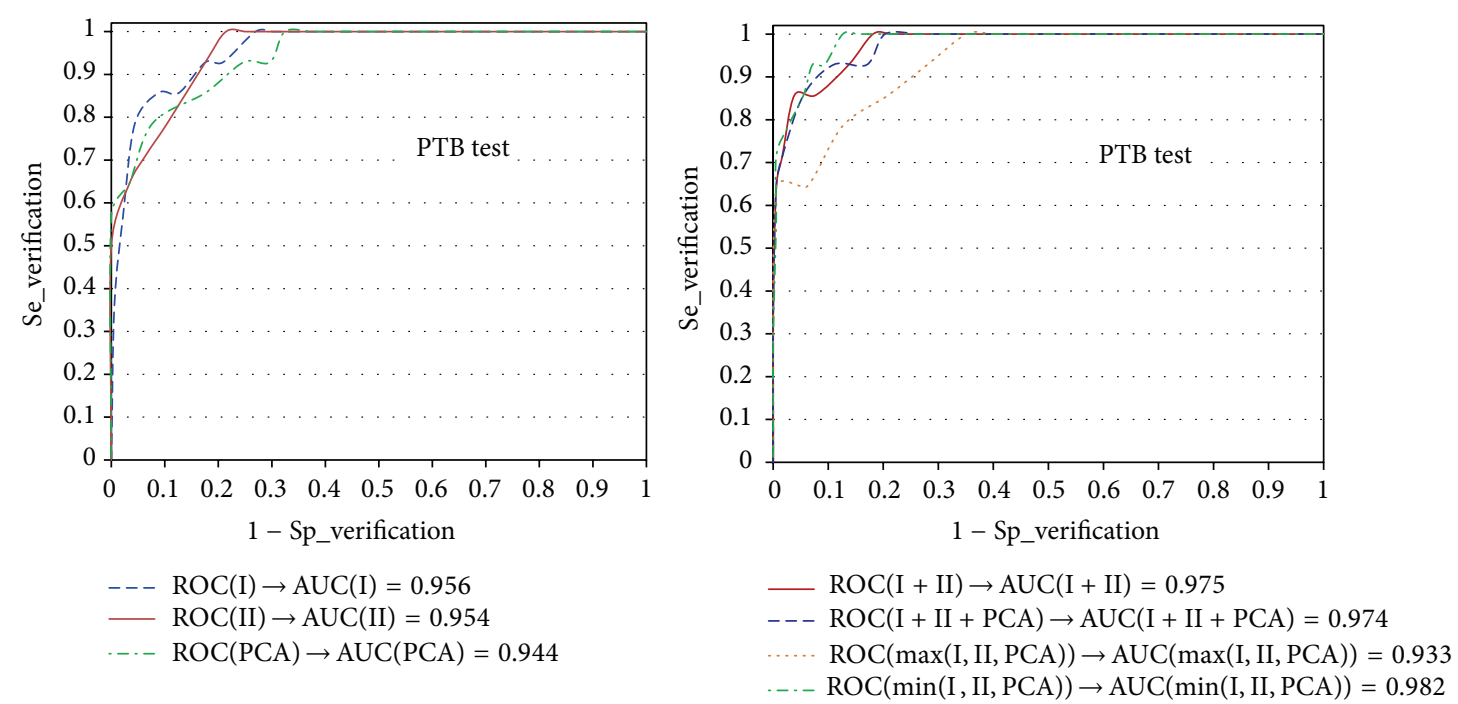

(b)

FIGURE 3: ROC curves and calculated AUCs for the test datasets: (a) ILSA test dataset (healthy subjects), (b) PTB healthy controls. 
TABLE 4: Sensitivity and specificity for person verification and accuracy for person identification over the test PTB dataset $(N=14$ subjects).

\begin{tabular}{|c|c|c|c|}
\hline & Se_verification & Sp_verification & AccID \\
\hline$r_{\text {I }}$ & $85.7 \%(12 / 14)$ & $91.2 \%(166 / 182)$ & $\begin{array}{c}92.9 \% \\
(13 / 14)\end{array}$ \\
\hline$r_{\text {II }}$ & $100 \%(14 / 14)$ & $69.8 \%(127 / 182)$ & $\begin{array}{c}92.9 \% \\
(13 / 14)\end{array}$ \\
\hline$r_{\mathrm{PCA}}$ & $92.9 \%(13 / 14)$ & $75.3 \%(137 / 182)$ & $\begin{array}{l}78.6 \% \\
(11 / 14)\end{array}$ \\
\hline$\left(r_{\mathrm{I}}+r_{\mathrm{II}}\right) / 2$ & $100 \%(14 / 14)$ & $\mathbf{8 1 . 9} \%(149 / 182)$ & $\begin{array}{l}92.9 \% \\
(13 / 14)\end{array}$ \\
\hline$\left(r_{\mathrm{I}}+r_{\mathrm{II}}+r_{\mathrm{PCA}}\right) / 3$ & $\mathbf{9 2 . 9} \%(13 / 14)$ & 83.0\% $(151 / 182)$ & $\begin{array}{l}\mathbf{8 5 . 7} \% \\
(12 / 14)\end{array}$ \\
\hline $\max \left(r_{\mathrm{I}}, r_{\mathrm{II}}, r_{\mathrm{PCA}}\right)$ & $78.6 \%(11 / 14)$ & $87.4 \%(159 / 182)$ & $\begin{array}{l}78.6 \% \\
(11 / 14)\end{array}$ \\
\hline $\min \left(r_{\mathrm{I}}, r_{\mathrm{II}}, r_{\mathrm{PCA}}\right)$ & $92.9 \%(13 / 14)$ & $90.7 \%(165 / 182)$ & $\begin{array}{l}92.9 \% \\
(13 / 14) \\
\end{array}$ \\
\hline
\end{tabular}
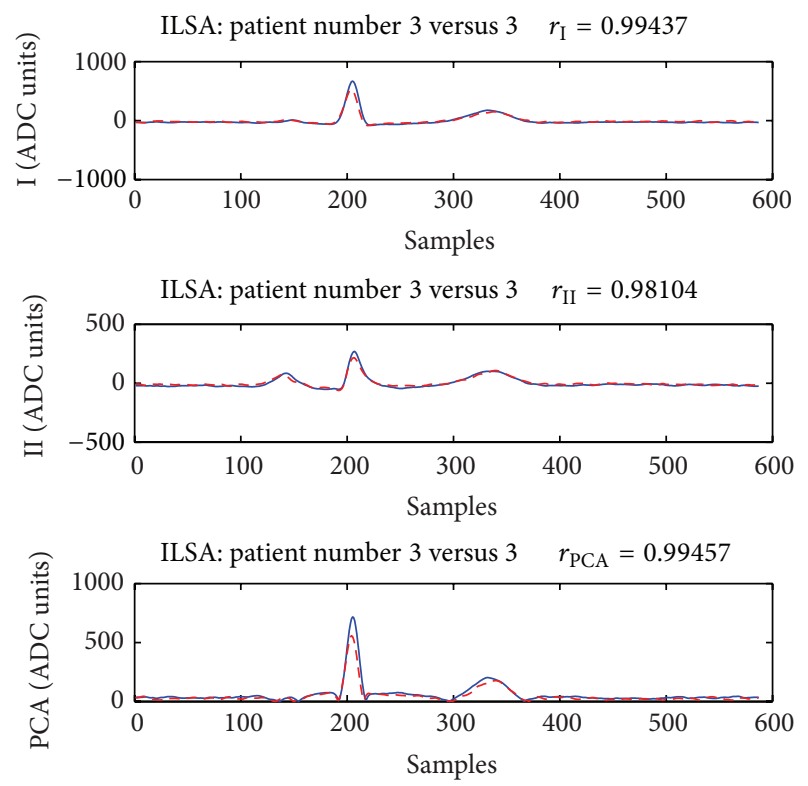

FIGURE 4: Subject 3 from the ILSA database. The strong correlation $(>0.98)$ between the P-QRS-T waveforms of this person of the training set in $T_{1}$ (blue solid line) and $T_{2}$ (red dashed line) assures both correct verification and correct identification. The time interval between the recordings of ECG in $T_{1}$ and $T_{2}$ is about 5 years.

values for $r_{\mathrm{I}}, r_{\mathrm{II}}, r_{\mathrm{PCA}},\left(r_{\mathrm{I}}+r_{\mathrm{II}}\right) / 2,\left(r_{\mathrm{I}}+r_{\mathrm{II}}+r_{\mathrm{PCA}}\right) / 3, \max \left(r_{\mathrm{I}}\right.$, $\left.r_{\mathrm{II}}, r_{\mathrm{PCA}}\right)$, and $\min \left(r_{\mathrm{I}}, r_{\mathrm{II}}, r_{\mathrm{PCA}}\right)$ depending on the application. Lower thresholds are suitable when low rejection rate (high sensitivity) is required at the expense of increased erroneous verifications. On the other side, the higher threshold values guarantee high security combined with higher rejection rate. Depending on the exact task that has to be solved, one can decide threshold values of the applied correlation coefficients. The ROC curves and AUCs presented in Figures 1(d) and 2(e) illustrate the behavior of the models over the train dataset.
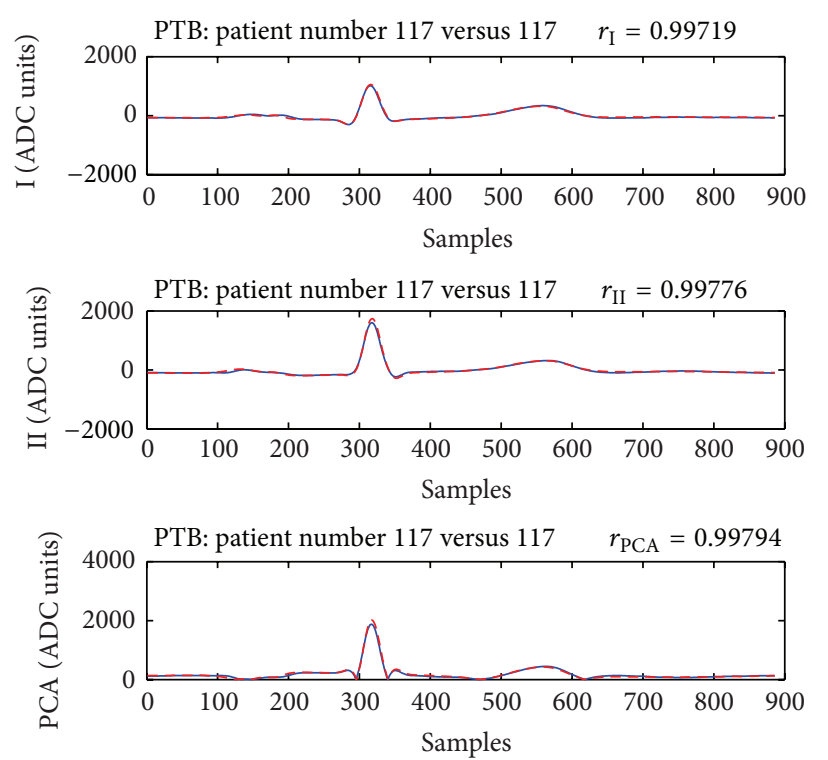

Figure 5: Subject 117 from the PTB database. The strong correlation (>0.99) between the P-QRS-T waveforms of this person in $T_{1}$ (blue solid line) and $T_{2}$ (red dashed line) assures both correct verification and correct identification. The time interval between the recordings of ECG in $T_{1}$ and $T_{2}$ is less than 24 hours.

According to [22], AUC higher than 0.9 is an approximate indication of an excellent classifier.

The person identification is performed by computing the correlation between the ECG of the subject under examination and a previously collected ECG database. The tested person is identified according to the maximal correlation to a subject in the database.

Considering the accuracy results (Tables 1, 2, and 3) over the training and test ILSA database and the AUCs (Figures 1(d) and 3), $r_{\text {II }}$ seems more reliable for person verification than $r_{\mathrm{I}}$. However, this observation is not confirmed by the results over the test PTB database (Table 4, Sp_verification < $70 \%)$. Although the first PCA component presents a combination between leads I and II, $r_{\mathrm{PCA}}$ do not lead to verification accuracy increase neither for the training nor for the test databases. Generally, the best verification/identification accuracy is achieved with the combined correlation coefficients $\left(r_{\mathrm{I}}+r_{\mathrm{II}}\right) / 2,\left(r_{\mathrm{I}}+r_{\mathrm{II}}+r_{\mathrm{PCA}}\right) / 3$ that also present AUCs higher than 0.9 (Figure 2(e) for training, Figure 3 for testing). This is in concord with the results of Poree et al. [7], who reported accuracy increase when more ECG leads are involved in the analysis.

Considering the verification/identification of healthy persons, the following observations can be pointed out:

(i) There is comparable verification accuracy for both training and test part of ILSA database (Table 1 versus Table 2, AUCs in Figures 1(d) and 2(e) versus Figure 3).

(ii) The identification accuracy is higher in the healthy ECG-ILSA test set $(N=49$ Table 2$)$ than in the learning set $(N=98$, Table 1$)$. 

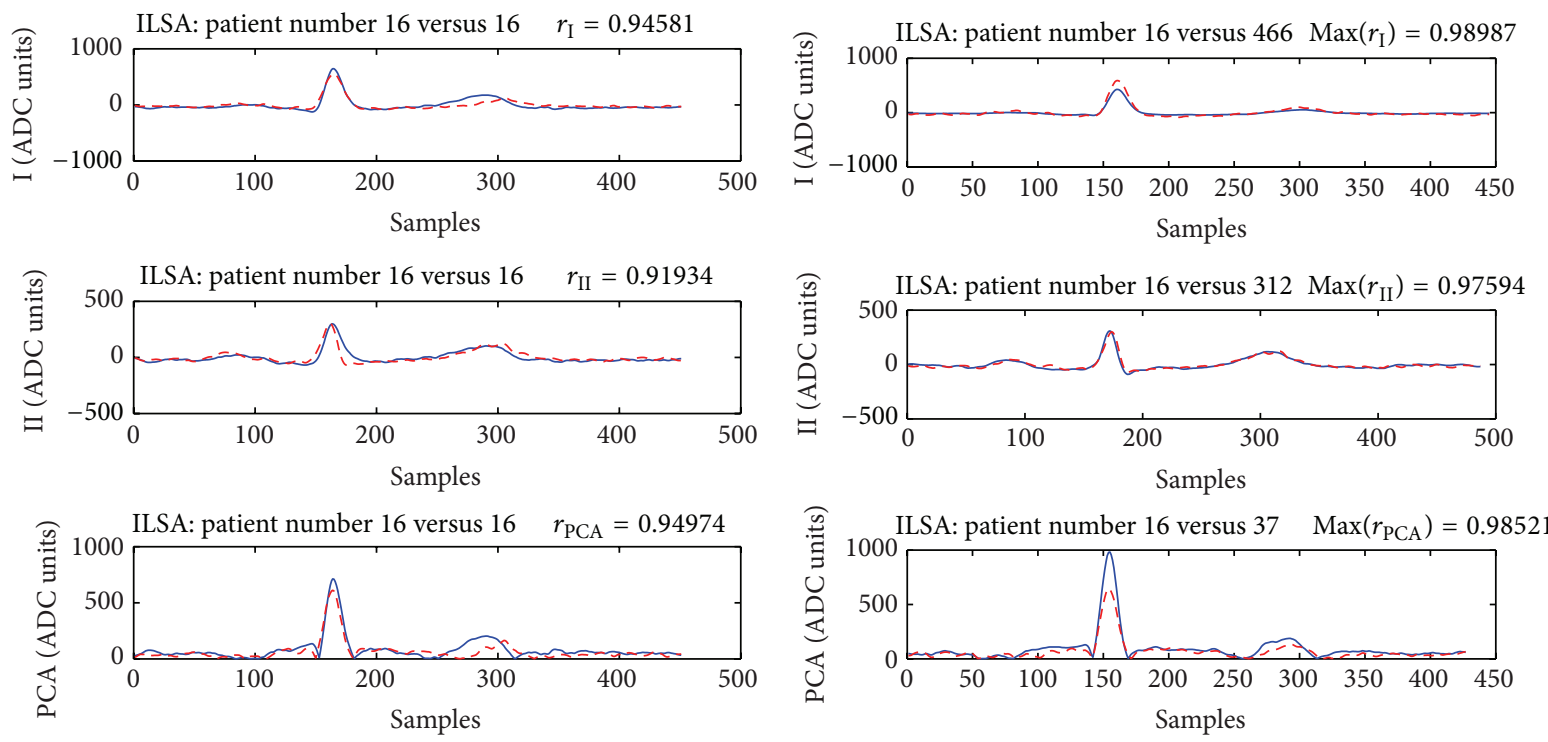

(a)

(b)

FIGURE 6: Subject 16 from the test part of ILSA database. (a) The assessed correlation coefficients $r_{\mathrm{I}}<0.946, r_{\mathrm{II}}=0.919$, and $r_{\mathrm{PCA}}<0.949$ are below the preset threshold values $\left(0.96,0.92\right.$, and 0.95 , resp.) and the identity of this person will not be verified neither by means of $r_{\mathrm{I}}, r_{\mathrm{II}}$, and $r_{\mathrm{PCA}}$ nor by applying any of their combinations. (b) This subject will be identified as subjects $466\left(\operatorname{Max}\left(r_{\mathrm{I}}\right) \sim 0.99, \max \left(r_{\mathrm{I}}, r_{\mathrm{II}}, r_{\mathrm{PCA}}\right)=\right.$ 0.99); $312\left(\operatorname{Max}\left(r_{\mathrm{II}}\right) \sim 0.98\right), 37\left(\operatorname{Max}\left(r_{\mathrm{PCA}}\right) \sim 0.99\right) ; 134\left(\left(r_{\mathrm{I}}+r_{\mathrm{II}}\right) / 2=0.95,\left(r_{\mathrm{I}}+r_{\mathrm{II}}+r_{\mathrm{PCA}}\right) / 3=0.96\right)$; or $439\left(\min \left(r_{\mathrm{I}}, r_{\mathrm{II}}, r_{\mathrm{PCA}}\right)=0.94\right)$. When the patient's ECG is compared to his own previously recorded ECG $\left(r_{\mathrm{I}}+r_{\mathrm{II}}\right) / 2=0.93,\left(r_{\mathrm{I}}+r_{\mathrm{II}}+r_{\mathrm{PCA}}\right) / 3=0.94, \max \left(r_{\mathrm{I}}, r_{\mathrm{II}}, r_{\mathrm{PCA}}\right)=0.95$, and $\min \left(r_{\mathrm{I}}, r_{\mathrm{II}}, r_{\mathrm{PCA}}\right)=0.92$.
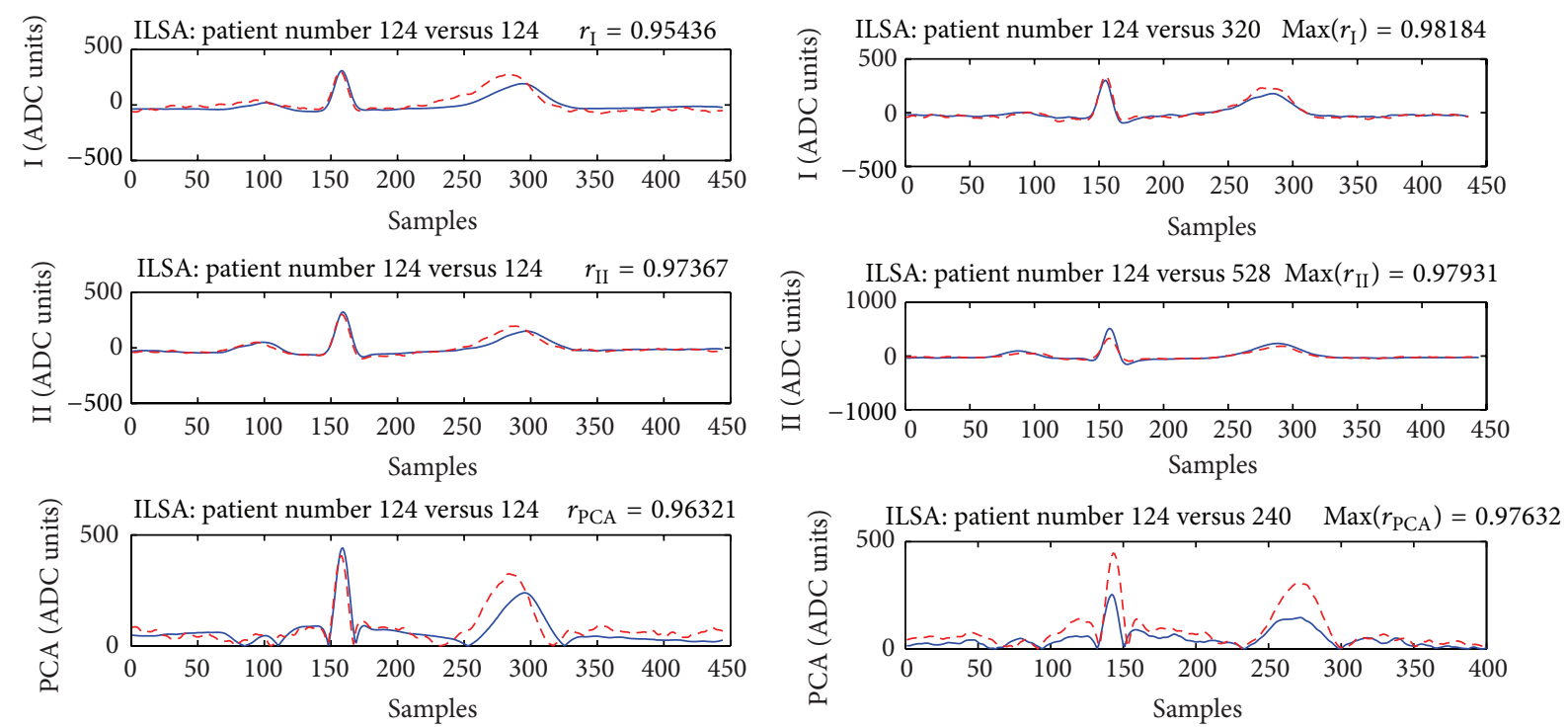

(a)

(b)

FIGURE 7: Subject 124 from the training part of ILSA database. Considering the independent correlation coefficients $r_{\mathrm{I}}, r_{\mathrm{II}}$, and $r_{\mathrm{PCA}}$, this subject will be identified either as subjects $320\left(\operatorname{Max}\left(r_{\mathrm{I}}\right)=0.98, \max \left(r_{\mathrm{I}}, r_{\mathrm{II}}, r_{\mathrm{PCA}}\right)=0.98\right), 528\left(\operatorname{Max}\left(r_{\mathrm{II}}\right)=0.979\right), 240\left(r_{\mathrm{PCA}}=0.98\right)$ or as subject $255\left(\min \left(r_{\mathrm{I}}, r_{\mathrm{II}}, r_{\mathrm{PCA}}\right)=0.96\right)$. However, the combined correlation coefficients $\left(r_{\mathrm{I}}+r_{\mathrm{II}}\right) / 2,\left(r_{\mathrm{I}}+r_{\mathrm{II}}+r_{\mathrm{PCA}}\right) / 3$ both have maximal values of 0.96 when the ECG in $T_{2}$ of this person is compared to his own ECG in $T_{1}$. 

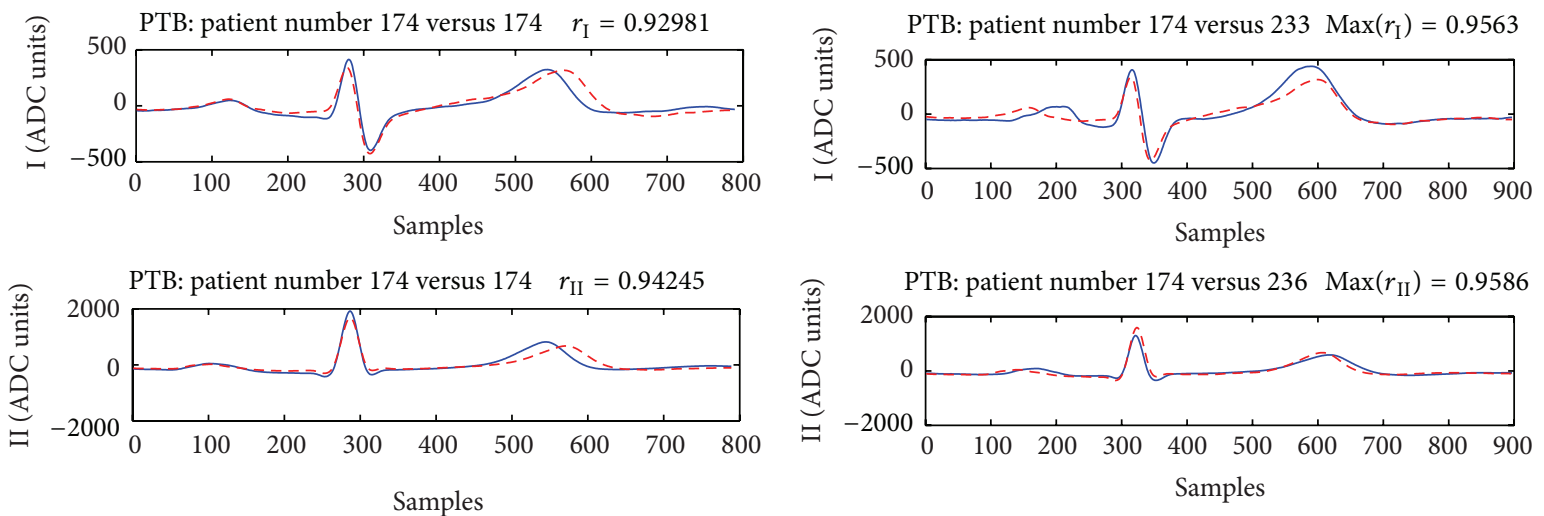

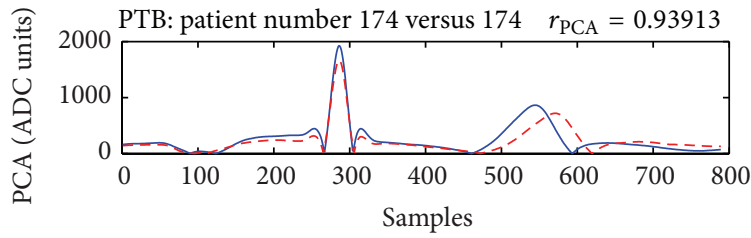

(a)

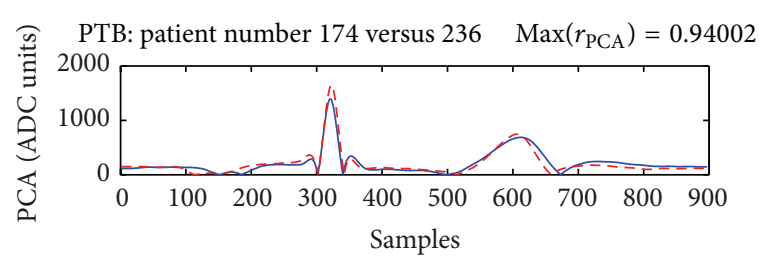

(b)

FIGURE 8: Subject 174 from the PTB database-time interval between the recordings of ECG in $T_{1}$ and $T_{2}$ is 59 days. (a) The assessed correlation coefficients $r_{\mathrm{I}}=0.93$ and $r_{\mathrm{PCA}}=0.94$ are below the preset threshold values (0.96 and 0.95, resp.) and the identity of this person will not be verified. (b) This subject will be identified as 233 according to $\operatorname{Max}\left(r_{\mathrm{I}}\right) \sim 0.96,\left(r_{\mathrm{I}}+r_{\mathrm{II}}\right) / 2=0.95,\left(r_{\mathrm{I}}+r_{\mathrm{II}}+r_{\mathrm{PCA}}\right) / 3=0.95, \min \left(r_{\mathrm{I}}, r_{\mathrm{II}}, r_{\mathrm{PCA}}\right)=$ 0.94 , or as 236 if $\operatorname{Max}\left(r_{\mathrm{II}}\right) \sim 0.96, \operatorname{Max}\left(r_{\mathrm{PCA}}\right) \sim 0.97$, and $\max \left(r_{\mathrm{I}}, r_{\mathrm{II}}, r_{\mathrm{PCA}}\right)=0.97$ are considered. When the patient's ECG is compared to his own previously recorded ECG $\left(r_{\mathrm{I}}+r_{\mathrm{II}}\right) / 2=0.94,\left(r_{\mathrm{I}}+r_{\mathrm{II}}+r_{\mathrm{PCA}}\right) / 3=0.94, \max \left(r_{\mathrm{I}}, r_{\mathrm{II}}, r_{\mathrm{PCA}}\right)=0.94$, and $\min \left(r_{\mathrm{I}}, r_{\mathrm{II}}, r_{\mathrm{PCA}}\right)=0.93$.

(iii) There is lower identification accuracy for the test ILSA dataset when all ECGs in the ECG-ILSA test set $(N=$ 442) are used as database for comparison (Table 3 versus Tables 1 and 2).

(iv) There is higher verification/identification accuracy for the test PTB dataset compared to the ECG-ILSA training and test sets.

These observations can be motivated by the following remarks:

(i) The better verification accuracy for the test PTB dataset can be explained by the shorter temporal interval between the two acquisition times $T_{1}$ and $T_{2}$, compared to the temporal interval of 5 years in the ECG_ILSA database which is in concord with the observations in [14]. In fact the 14 subjects are characterized by
(a) $\left(T_{2}-T_{1}\right)<24$ hours in 7 cases,
(b) $\left(T_{2}-T_{1}\right)<1$ month in 2 patients,
(c) $\left(T_{2}-T_{1}\right)>1$ and $<3$ months in 2 patients,
(d) $\left(T_{2}-T_{1}\right)>6$ months for 3 patients.

(ii) The identification accuracy is influenced by the number of records in the testing set, and, consequently with reduced number of patients, it is possible to obtain higher values. In fact, the PTB database with only 14 records produces better identification accuracy $\left(92.9 \%\right.$ with $\left(r_{\text {I }}+r_{\text {II }}\right) / 2$ in Table 4$)$ if compared with the test set of 49 healthy people of the ECG-ILSA database (Table 2) or if compared with all 442 subjects of the test set (Table 3). This behavior is in agreement with the study of Zokaee and Faez [10] where the increasing from 10 to 50 of the number of ECGs in the test dataset produced decreasing of the accuracy of about $10 \%$ (from $98.6 \%$ to $89 \%$ ).

In literature there are only few studies [5] which consider the ECG biometric recognition in the presence of cardiac irregularity conditions, although they were performed mainly with single day sessions. Consequently, the present work represents a significant test on the influence of the personal health status for the verification/identification accuracy in the presence of long term interval recordings.

Our observations for cardiac and hypertension persons are as follows.

Considering the $\left(r_{\mathrm{I}}+r_{\mathrm{II}}\right) / 2$ classification method, the validation procedure on the entire test set of 442 healthy and nonhealthy patients, some observations can be performed as follows:

(i) The hypertension group (Table 3 ) shows lower Se_verification (82.6\%) and AccID (53.7\%) compared to the healthy and cardiac groups (resp., $89.8 \%, 86.3 \%$ and $59.2 \%, 60.6 \%)$.

(ii) The cardiac group shows a slight improvement in AccID (60.6\%) and in Sp_verification (91.8\%) in comparison with the healthy group (59.2\% and $86.6 \%)$.

These results show the robustness of the proposed classification method for person identification and verification, 
TABLE 5: Comparison between verification/identification accuracy achieved by the proposed method over the test dataset and the results reported by other authors with different databases used (db). The number of ECG recordings per patient (1 rpp for one and mrpp for more) and the acquisition interval (acq_int) on the same patient are reported.

\begin{tabular}{|c|c|c|}
\hline Method & Database & Accuracy \\
\hline Agrafioti and Hatzinakos, 2009 [5] & $\begin{array}{l}\text { MIT-BIH normal sinus } \\
\text { MIT-BIH arrhythmia } 1 \mathrm{rpp} \\
\text { PTB db, } 13 \text { healthy subjects mrpp }\end{array}$ & $\begin{array}{c}\text { AccID }=96.2 \% \\
\text { Sp_ver }=99 \% \\
\text { Se_ver }=87 \%\end{array}$ \\
\hline Israel et al., 2005 [3] & $\begin{array}{l}\text { Own db: } 29 \text { subjects } \\
\text { close in time recordings }\end{array}$ & AccID = $100 \%$ \\
\hline Lourenço et al., 2011 [13] & $\begin{array}{l}\text { Own db: } 16 \text { subjects } \\
\text { close in time recordings }\end{array}$ & $\begin{array}{l}\text { AccID }=94.3 \% \\
\text { Se }=\text { Sp }=87 \%\end{array}$ \\
\hline Sidek et al., 2012 [11] & Own db: 30 healthy subj., close in time recordings & AccID $=96.1 \%$ \\
\hline Wang et al., 2008 [4] & $\begin{array}{l}\text { MIT-BIH normal sinus } 1 \mathrm{rpp} \\
\text { PTB db, } 13 \text { healthy subj. mrpp }\end{array}$ & AccID $=100 \%$ \\
\hline Zhao et al., 2013 [1] & $\begin{array}{l}\text { MIT-BIH ST change db, long-term ST db } 1 \mathrm{rpp} \text {; } \\
\text { PTB db, } 12 \text { healthy subj. mrpp }\end{array}$ & $\begin{array}{l}\text { AccID }(\text { tot })=95.6 \% \\
\text { AccID }(\text { PTB })=96 \%\end{array}$ \\
\hline Zokaee and Faez, 2012 [10] & $\begin{array}{l}\text { MIT-BIH db } 1 \text { rpp } \\
\text { Own Holter, } 50 \text { subjects } 1 \text { rpp }\end{array}$ & $\begin{array}{l}\text { AccID }=100 \% \\
\text { AccID }=89 \%\end{array}$ \\
\hline Poree et al., 2011 [7] & Own db: 11 subjects, mrpp, acq_int $=16$ months & AccID $=91.4 \%$ \\
\hline Lee et al., 2012 [23] & Own db: 10 subjects, $\sim 100 \mathrm{rpp}$ within 3-month period & AccID $=99.5 \%$ \\
\hline Wübbeler et al., 2007 [8] & $\mathrm{db}$ from 74 subjects, mrpp, acq_int $=16$ months & $\begin{array}{l}\text { AccID }=98.1 \% \\
\mathrm{Se}=\mathrm{Sp}=97.2 \%\end{array}$ \\
\hline \multirow{2}{*}{$\begin{array}{l}\text { Our method } \\
\text { (based on assessment of }\left(r_{\mathrm{I}}+r_{\mathrm{II}}\right) / 2 \text { ) }\end{array}$} & Test ILSA db, 49 healthy subjects mrpp & $\begin{array}{l}\text { AccID }=77.6 \% \\
\text { Se_ver }=89.8 \% \\
\text { Sp_ver }=83.9 \%\end{array}$ \\
\hline & Test PTB db, 14 healthy subjects mrpp & $\begin{aligned} \text { AccID } & =92.9 \% \\
\text { Se_ver } & =100 \% \\
\text { Sp_ver } & =81.9 \%\end{aligned}$ \\
\hline
\end{tabular}

although the obtained results are probably not effective for real applications. It is interesting to consider the classification of some examples with or without problematic identification/verification.

The example in Figure 5 shows strong correlation between 2 ECG recordings of one subject from the test database that lead to unconditional correct person verification and identification. It should be mentioned, however, that both ECGs are recorded within time interval less than 24 hours. High values of the correlation coefficients are also observed for ECG recordings with temporal interval of several years (see the example in Figure 4); however, for such cases the expected ECG changes are visible.

On the other pole, the examples in Figures 6 and 8 present aligned P-QRS-T segments of subjects, for whom most of the correlation coefficients are below the thresholds for person verification. Moreover, the ECGs of these people are more correlated with ECGs of other members in the datasets used for comparison which leads to incorrect identification.

The example in Figure 7 proves the advantages of the combined correlation coefficients $\left(r_{\mathrm{I}}+r_{\mathrm{II}}\right) / 2$ and $\left(r_{\mathrm{I}}+\right.$ $\left.r_{\text {II }}+r_{\text {PCA }}\right) / 3$ that prevent erroneous identifications when strong correlation with a wrong ECG from the database for comparison is observed only in lead I, lead II, or the first PCA component.

Considering the differences in the verification/identification accuracy over the training and test databases, as well as the presented examples for correct and erroneous verification, we can conclude that the changes appearing in ECG with time could affect the accuracy of person verification/identification. This is also confirmed by the comparison between our results on healthy persons and the results reported in literature (see Table 5). It is obvious that the studies using only close in time ECG recordings $[3,10,11]$ report higher identification and/or verification performances. The direct comparison to studies that use the healthy controls in PTB database is not possible, since the authors have applied their methods on part of the healthy persons (12 or 13 from all 14 patients) and have mixed them with cases that do not have separated in time ECGs. Nevertheless, our results could be positively compared with studies for the identification task which consider ECG datasets with comparable size and similar temporal interval between the 1st and the 2nd recording $[1,5,7,10]$.

The ECG variability within small time interval could be due to electrode position variation between the two recordings. Even a meal can cause considerable ECG changes, both in healthy people and in cardiac patients. ECG recordings acquired one or more years apart show larger intraindividual variability. Sources such as age, weight, and heart position then come into play, in addition to the sources already having effect on smaller time scales. The influences of different factors on the intraindividual ECG variability are summarized by Schijvenaars [24]. In healthy people, the most prominent changes after a standard meal are an increase of heart rate, a decrease of T-wave amplitude and QT interval, and small 
left axis shifts of the QRS and T-axes. The influences of age, weight, and heart position are often interdependent; the heart position becomes more horizontal when one gains weight, people generally gain some weight as years pass, and so forth. The general trend is a decrease in amplitudes and a left axis shift in frontal QRS axis with increasing age or weight. The general age trends found among adults are decrease of precordial amplitudes (QRS spatial magnitude decreases with approximately $8 \%$ per decade), a leftward shift of the frontal plane axis (approximately $10^{\circ}$ per decade), and a more anterior axis in the horizontal plane. Interval durations' increase for PR and QT interval and decrease in QRS duration are also observed.

Considering the above mentioned sources of ECG variability, as well as our observations over the training and test databases, we conclude the following.

(i) Aiming at higher identification accuracy, the database for comparison (TrainSet1, TestSet1) should be kept as small as possible for the particular application and should be updated with actual ECG recordings.

(ii) Aiming at higher verification accuracy, the ECG recordings in the database for comparison should be updated on a regular basis (as short as possible, e.g., every time when the person passes through border control). The new ECG could replace the old one, after verification, or could be added to a personal folder with a reasonable size. This would guarantee higher values of Se_verification and would provide the opportunity to increase the threshold values for the correlation coefficients which in turn would increase Sp_verification.

\section{Conclusions}

This paper studies the reliability of the ECG signal for person verification/identification. The population based ECG-ILSA database of 540 patients (147 healthy subjects, 175 patients with cardiac diseases, and 218 with hypertension) has been considered for the validation procedure. For a more robust validation procedure, considering the temporal variability of ECG signal and/or modifications in the pathologies, the learning phase was performed only in the healthy group, and the testing procedures have been performed also with nonhealthy patients. The proposed method relies on assessment of correlation coefficients as well as their linear and nonlinear combinations and provides $100 \%$ verification sensitivity combined with $18.1 \%$ erroneous verification rate in the PTB database, a widely used test set in literature, with a relatively short temporal interval of ECG acquisition and a limited number of healthy subjects $(N=14)$. This dataset produces an identification accuracy of $92.9 \%$. The test set of healthy subjects in the ECG-ILSA database $(N=49)$ produces a lower verification sensitivity (89.8\%) and identification accuracy $(77.6 \%)$ but a better erroneous verification rate (16.1\%). For cardiac and hypertension patients we observe decreased sensitivity and increased specificity for verification. Considering the identification task, our conclusions are that the accuracy depends generally on the size of the database for comparison, but not on the person's health status.

Although the ECG is considered to be strongly individual biometric feature, this study shows that there are some changes over time that could prevent correct individual verification, and two healthy persons could have similar ECGs that could lead to incorrect identification. This poses requirements towards the database stored for comparison, such as size of the database and maximal time interval between the tested ECG and the ECG stored in this database. Despite these limitations, the ECG has the indisputable advantage to be not susceptible to falsification. It seems to be a reliable biometric characteristic for specific access control applications, which operate with smaller databases for comparison.

Although there are several limitations in this methodology, which can prevent its use in real practice, it could be possible to overcome the drawbacks with the inclusion of some demographic/personal information in the classification process for obtaining "certain" identification. Moreover, this study proves the potential of ECG application for increasing the reliability of person verification and identification based on biometrical information from other sources.

\section{Conflict of Interests}

The authors declare that there is no conflict of interests regarding the publication of this paper.

\section{Acknowledgment}

This study is supported by Bulgarian National Science Fund, Grant no. T02/11.

\section{References}

[1] Z. Zhao, L. Yang, D. Chen, and Y. Luo, "A human ECG identification system based on ensemble empirical mode decomposition," Sensors, vol. 13, no. 5, pp. 6832-6864, 2013.

[2] L. Biel, O. Pettersson, L. Philipson, and P. Wide, "ECG analysis: a new approach in human identification," IEEE Transactions on Instrumentation and Measurement, vol. 50, no. 3, pp. 808-812, 2001.

[3] S. A. Israel, J. M. Irvine, A. Cheng, M. D. Wiederhold, and B. K. Wiederhold, "ECG to identify individuals," Pattern Recognition, vol. 38, no. 1, pp. 133-142, 2005.

[4] Y. Wang, F. Agrafioti, D. Hatzinakos, and K. N. Plataniotis, "Analysis of human electrocardiogram for biometric recognition," EURASIP Journal on Advances in Signal Processing, vol. 2008, Article ID 148658, 2008.

[5] F. Agrafioti and D. Hatzinakos, "ECG biometric analysis in cardiac irregularity conditions," Signal, Image and Video Processing, vol. 3, no. 4, pp. 329-343, 2009.

[6] K. N. Plataniotis, D. Hatzinakos, and J. K. M. Lee, "ECG biometric recognition without fiducial detection," in Proceedings of the Biometrics Symposium: Special Session on Research at the Biometric Consortium Conference (BSYM '06) , 2006 (BSYM '06), pp. 1-6, Baltimore, Md, USA, September 2006.

[7] F. Poree, A. Gallix, and G. Carrault, "Biometric identification of individuals based on the ECG. Which conditions?" in 
Proceedings of the Computing in Cardiology, vol. 38, pp. 761-764, IEEE, Hangzhou, China, September 2011.

[8] G. Wübbeler, M. Stavridis, D. Kreiseler, R.-D. Bousseljot, and C. Elster, "Verification of humans using the electrocardiogram," Pattern Recognition Letters, vol. 28, no. 10, pp. 1172-1175, 2007.

[9] C. Ye, M. T. Coimbra, and B. V. K. V. Kumar, "Investigation of human identification using two-lead Electrocardiogram (ECG) signals," in Proceedings of the 4th IEEE International Conference on Biometrics: Theory, Applications and Systems (BTAS '10), Washington, DC, USA, September 2010.

[10] S. Zokaee and K. Faez, "Human identification based on ECG and palmprint," International Journal of Electrical and Computer Engineering, vol. 2, no. 2, pp. 261-266, 2012.

[11] K. Sidek, I. Khalil, and M. Smolen, "ECG biometric recognition in different physiological conditions using robust normalized QRS complexes," in Proceedings of the Computers in Cardiology, pp. 97-100, IEEE, Kraków, Poland, September 2012.

[12] F. Poree, J. Y. Bansard, G. Kervio, and G. Carrault, "Stability analysis of the 12-lead ECG morphology in different physiological conditions of interest for biometric applications," in Proceedings of the Computers in Cardiology, vol. 36, pp. 285-288, IEEE, Park City, Utah, USA, September 2009.

[13] A. Lourenço, H. Silva, and A. Fred, "Unveiling the biometric potential of finger-based ECG signals," Computational Intelligence and Neuroscience, vol. 2011, Article ID 720971, 8 pages, 2011.

[14] I. Odinaka, P.-H. Lai, A. D. Kaplan, J. A. O'Sullivan, E. J. Sirevaag, and J. W. Rohrbaugh, "ECG biometric recognition: a comparative analysis," IEEE Transactions on Information Forensics and Security, vol. 7, no. 6, pp. 1812-1824, 2012.

[15] A. D. C. Chan, M. M. Hamdy, A. Badre, and V. Badee, "Wavelet distance measure for person identification using electrocardiograms," IEEE Transactions on Instrumentation and Measurement, vol. 57, no. 2, pp. 248-253, 2008.

[16] S. Maggi, M. Zucchetto, F. Grigoletto et al., "The Italian longitudinal study on aging (ILSA): design and methods," Aging Clinical and Experimental Research, vol. 6, no. 6, pp. 464-470, 1994.

[17] L. Amaducci, M. Baldereschi, A. DiCarlo et al., "Prevalence of chronic diseases in older Italians: comparing self-reported and clinical diagnoses," International Journal of Epidemiology, vol. 26, no. 5, pp. 995-1002, 1997.

[18] G. Bortolan, M. Bressan, and F. Golferini, "Serial analysis in the ECG-ILSA database: study of longitudinal modifications," in Proceedings of the Computers in Cardiology, vol. 26, pp. 667670, Hannover, Germany, September 1999.

[19] G. Bortolan, M. Bressan, F. Golferini, and ILSA Study Group, "QT dispersion in the elderly. The ILSA study," Aging Clinical and Experimental Research, vol. 16, no. 5, pp. 342-348, 2004.

[20] G. Bortolan, M. Bressan, and I. Christov, "Longitudinal modifications of T-loop morphology," in Proceedings of the Computers in Cardiology, vol. 29, pp. 685-688, IEEE, September 2002.

[21] I. I. Christov, "Real-time electrocardiogram QRS detection using combined adaptive threshold," Biomedical Engineering Online, vol. 3, no. 28, pp. 1-9, 2004.

[22] F. Gorunescu, Data Mining: Concepts, Models and Techniques, Springer, Beijing, China, 2011.

[23] J. Lee, Y. Chee, and I. Kim, "Personal identification based on vectorcardiogram derived from limb leads electrocardiogram," Journal of Applied Mathematics, vol. 2012, Article ID 904905, 12 pages, 2012.
[24] B. Schijvenaars, Intra-individual variability of electrocardiogram. Assessment and exploitation in computerized ECG analysis [Ph.D. thesis], Erasmus University Rotterdam, Rotterdam, The Netherlands, 2000. 

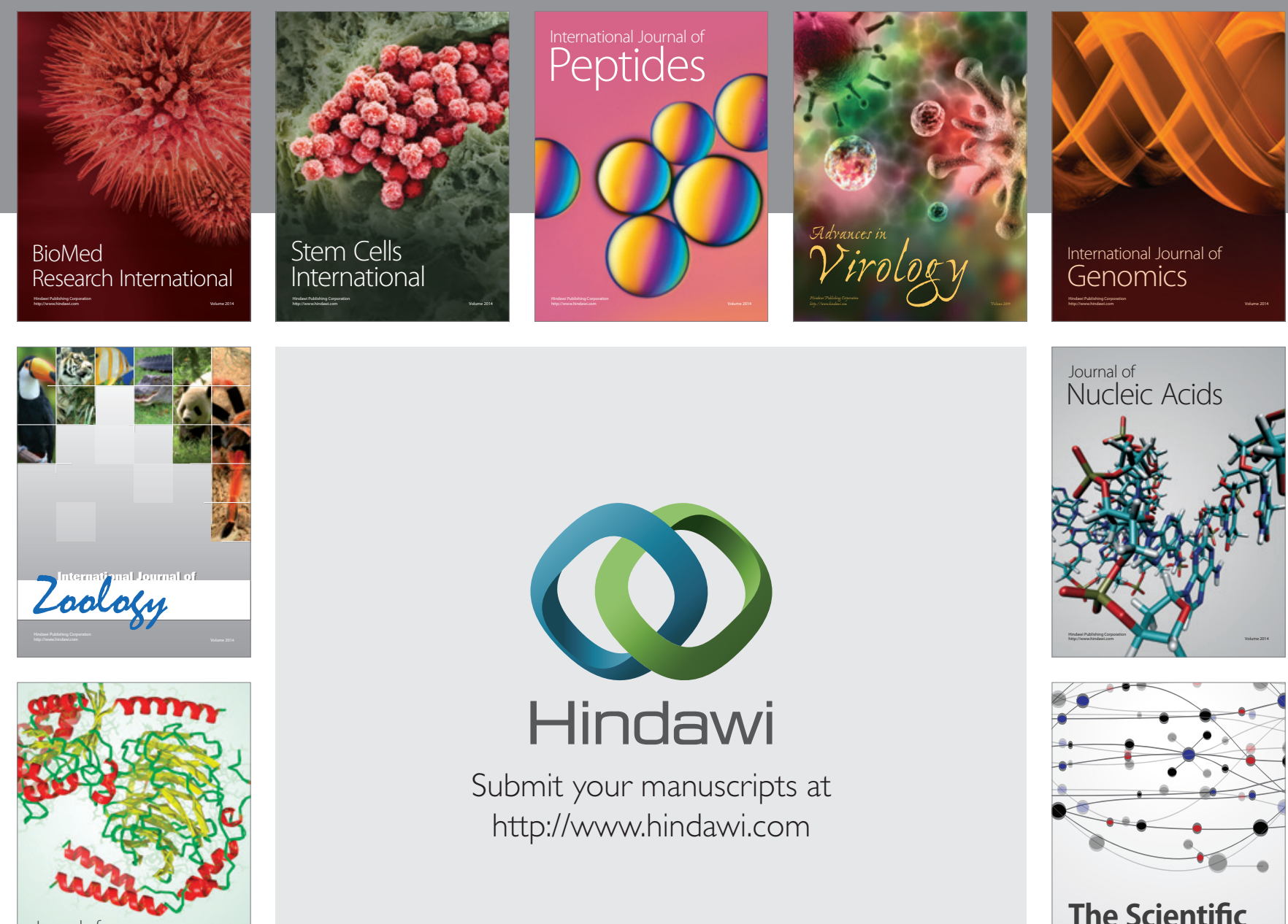

Submit your manuscripts at

http://www.hindawi.com

Journal of
Signal Transduction
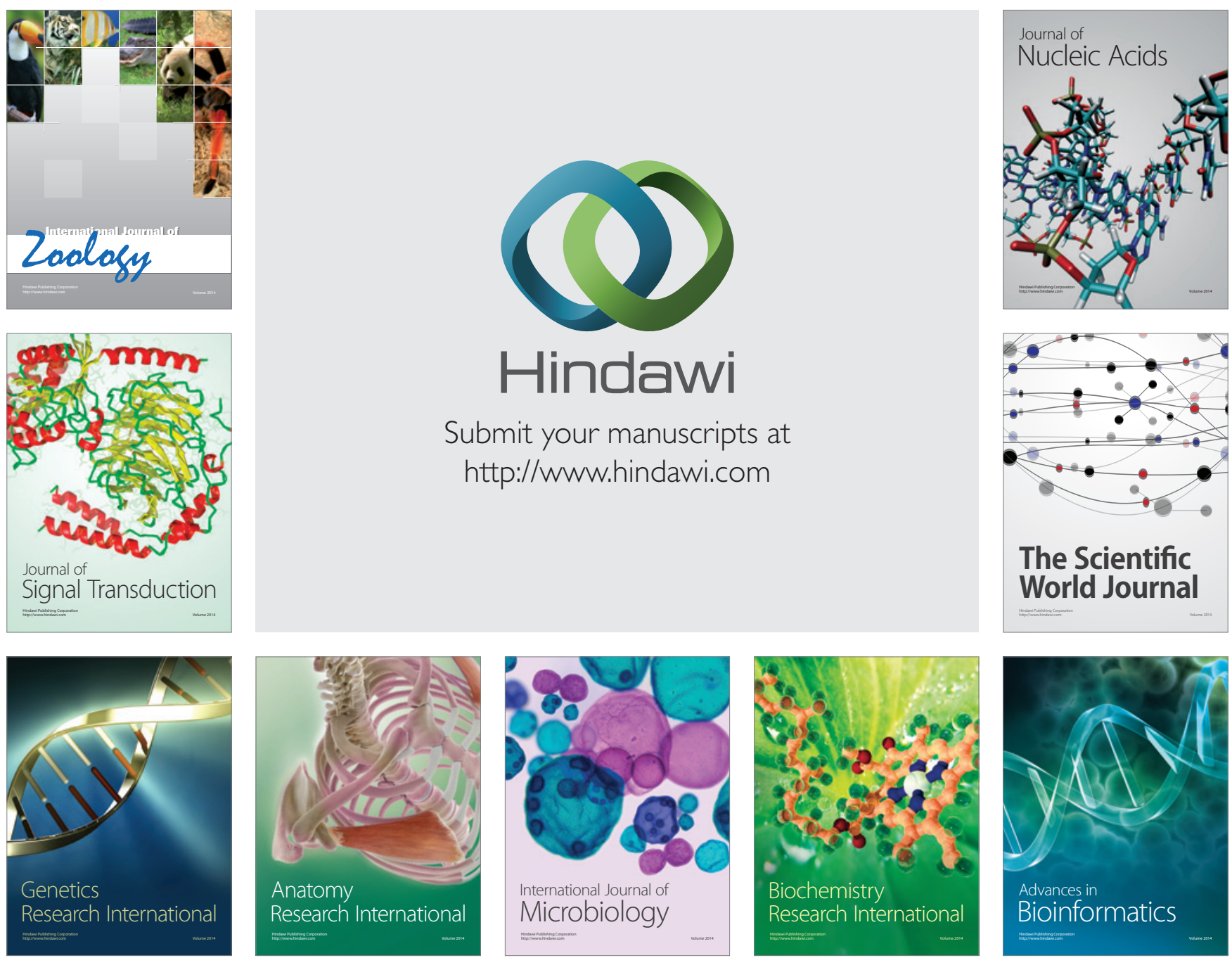

The Scientific World Journal
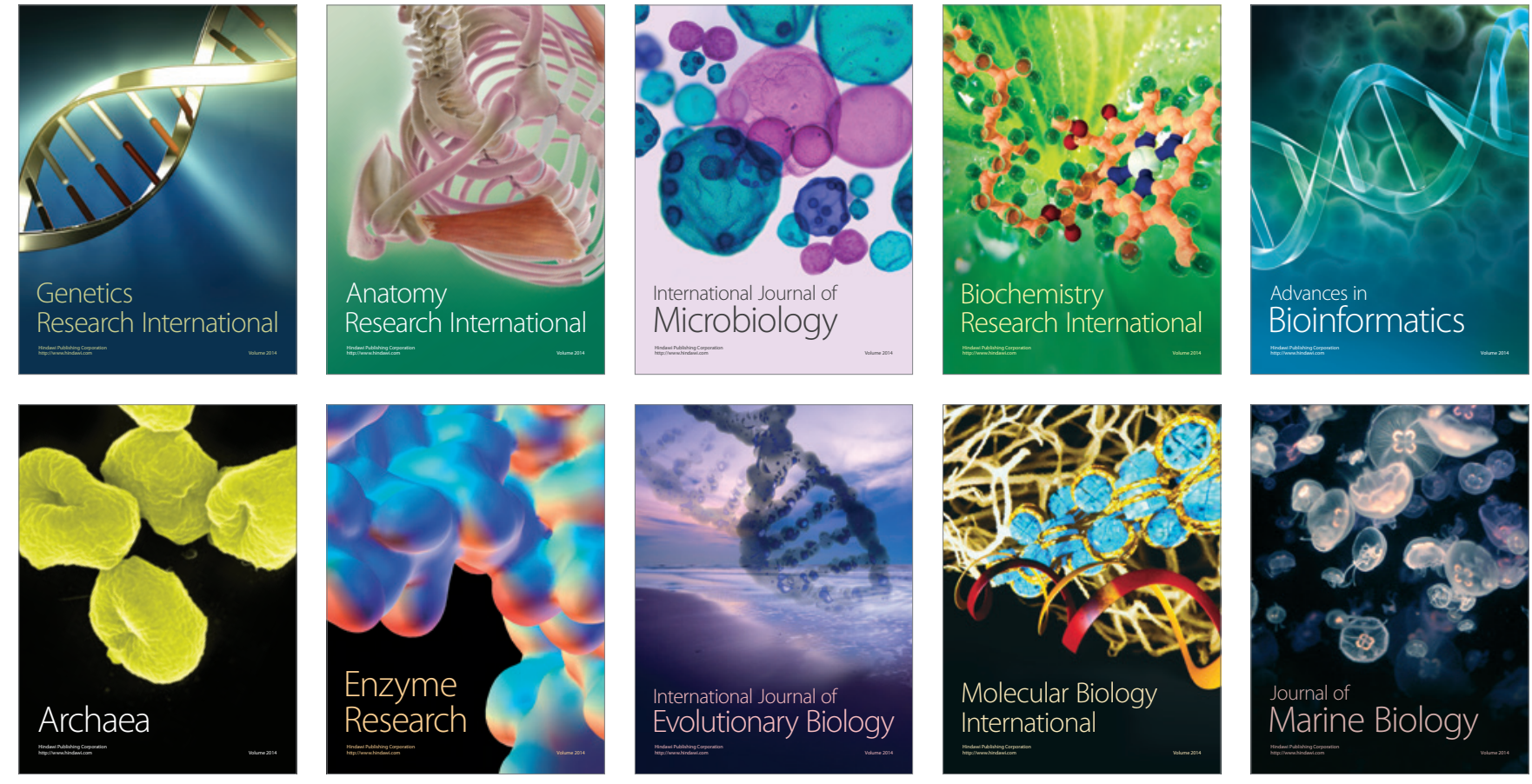